\title{
Wild-type measles virus is intrinsically dual-tropic
}

\author{
Makoto Takeda ${ }^{1}$, Maino Tahara ${ }^{1}$, Noriyo Nagata ${ }^{2}$ and Fumio Seki ${ }^{1}$ \\ 1 Department of Virology 3, National Institute of Infectious Diseases, Tokyo, Japan \\ ${ }^{2}$ Department of Pathology, National Institute of Infectious Diseases, Tokyo, Japan
}

\section{Edited by:}

Yasuko Yokota, National Institute of

Infectious Diseases, Japan

\section{Reviewed by:}

Masato Tsurudome, Mie University Graduate School of Medicine, Japan Bert Rima, Queen's University

Belfast, UK

\section{*Correspondence:}

Makoto Takeda, Department of Virology 3, National Institute of

Infectious Diseases, Gakuen 4-7-1,

Musashimurayama, Tokyo 208-0011,

Japan.

e-mail:mtakeda@nih.go.jp
Measles is a highly contagious disease that causes temporary and severe immunosuppression in patients. Signaling lymphocyte activation molecule (SLAM) expressed on cells of the immune system functions as a receptor for measles virus (MV). In addition to SLAM, vaccine strains of MV also use a ubiquitously expressed complement regulatory protein, CD46, as a receptor, whereas wild-type (wt) MV strains do not use this receptor. However, recent studies have indicated that SLAM is not the sole receptor for wt MV strains. These strains have an intrinsic ability to enter both immune and epithelial cells using distinct receptor binding sites in their hemagglutinin $(\mathrm{H})$ protein. Recently, a clear answer was obtained through the identification of an epithelial MV receptor, nectin4, expressed at adherens junctions, thereby greatly improving our knowledge of MV receptors. It is now clear that MV specifically targets two cell types, immune cells and epithelial cells, using SLAM and nectin4, respectively. MV loses the ability to use either SLAM or nectin4 when it possesses specific mutations in the H protein. However, nectin4-blind MV still infects SLAM-positive immune cells efficiently (SLAM-tropic), and conversely, SLAM-blind MV infects nectin4-positive epithelial cells efficiently (nectin4-tropic). In this regard, MV is intrinsically dual-tropic to immune cells and epithelial cells. Although many aspects and molecular mechanisms underlying immunosuppressive effects and a highly contagious nature of MV still remain to be elucidated, analyses of physiological functions of these two receptors would provide deep insights into MV pathogenesis.

Keywords: measles virus, dual-tropic, SLAM, nectin4, receptor

\section{MEASLES VIRUS}

Measles is a highly contagious acute viral disease characterized by high fever, malaise, coryza, conjunctivitis, cough, and a maculopapular rash (Griffin, 2007). Patients with measles develop a severe and temporary immunosuppression, which is often accompanied by secondary bacterial infections (Griffin, 2007). Despite the availability of highly effective vaccines, measles-related deaths were estimated to be 164,000 worldwide in 2008 (WHO, 2009). The causative agent is measles virus (MV), which belongs to the genus Morbillivirus in the family Paramyxoviridae. The virus particle is enveloped and contains a non-segmented negative-strand RNA genome encoding six tandem linked genes, $\mathrm{N}, \mathrm{P} / \mathrm{V} / \mathrm{C}, \mathrm{M}$, F, H, and L. The genome is encapsidated by the nucleocapsid (N) protein and associated with viral RNA-dependent RNA polymerases, forming a helical ribonucleoprotein complex (RNP). On the envelope, the viral particle possesses two types of viral glycoprotein spikes, the hemagglutinin $(\mathrm{H})$ and fusion $(\mathrm{F})$ proteins (Griffin, 2007). The H protein is responsible for binding to cellular receptors on the target host cells, and plays a key role in the determination of host cell specificity (tropism) of MV (Yanagi et al., 2009). Binding of the $\mathrm{H}$ protein to a receptor triggers $\mathrm{F}$ protein-mediated membrane fusion between the virus envelope and the host cell plasma membrane, releasing the RNP into the cytoplasm. In cells infected with $\mathrm{MV}$, the $\mathrm{H}$ and $\mathrm{F}$ proteins are expressed on the cell surface and cause cell-to-cell fusion, producing syncytia.

\section{DISCOVERIES OF CELLULAR RECEPTORS FOR MV}

Basically, MV specifically infects cells expressing its receptors. Therefore, the distribution pattern of its receptors is a key determinant of which cells become infected with MV (Yanagi et al., 2009). The initial discovery of an MV receptor came in 1993 (Dorig et al., 1993; Naniche et al., 1993). Two independent studies indicated that the receptor molecule for MV is the human membrane cofactor protein (MCP/CD46), a central component of the complement system, which is expressed ubiquitously on all organs and tissues throughout the human body (Dorig et al., 1993; Naniche et al., 1993). These findings were highly welcomed from the viewpoint that MV causes a systemic infection. Meanwhile, Kobune et al. (1990) reported the isolation of lymphotropic MV strains, and subsequent studies indicated that these lymphotropic MV strains do not use MCP/CD46 as a receptor (Yanagi et al., 2009). Importantly, Kobune's isolates exhibited a high virulence in experimentally infected monkeys, whereas $\mathrm{MCP} / \mathrm{CD} 46-$ using classical MV isolates caused no or mild disease in monkeys (Kobune et al., 1990, 1996; Takeda et al., 1998). Hence, new two questions have arisen for MV researchers. What is the receptor for these lymphotropic strains? Which strains are the real wild-type (wt) MV strains? In 2000, using Kobune's isolates, another receptor was identified (Tatsuo et al., 2000). This receptor is signaling lymphocyte activation molecule, also known as CD150 (SLAM/CD150), which is expressed on cells of the immune system (Tatsuo et al., 2000). Subsequent studies clarified 
that SLAM/CD150 is a receptor for wt MV strains circulating in patients, and that MCP/CD46 does not act as a receptor for wt MV strains (Yanagi et al., 2009). MCP/CD46 acts as a receptor only for vaccine and some laboratory MV strains (Yanagi et al., 2009). Currently, it is clear that these MV strains have acquired the ability to use MCP/CD46 as an alternative receptor to grow in laboratory cell lines lacking SLAM/CD150 expression (Yanagi et al., 2009).

Hence, it has become generally accepted that wt MV is a lymphotropic virus that specifically targets immune cells, similar to the case of human immunodeficiency virus (HIV) and human T cell lymphotropic virus type 1 (HTLV1). In 2000, a recombinant MV, IC323, was generated based on Kobune's first isolate (Takeda et al., 2000), and has greatly contributed to our understanding of the molecular bases for the pathogenesis of wt MV strains (Takeuchi et al., 2005; de Swart et al., 2007; Devaux et al., 2008, 2011; Leonard et al., 2008; Nakatsu et al., 2008; de Vries et al., 2010; Ludlow et al., 2010; Mühlebach et al., 2011; Noyce et al., 2011). At that time, only SLAM/CD150-positive cells were found to be susceptible to wt MV infections. However, it remained difficult to make a final conclusion that SLAM/CD150 is the sole receptor for wt $\mathrm{MV}$, because histopathological examinations of measles patients and monkeys infected with MV have revealed considerable levels of MV protein expression in the epithelia of various organs, and histopathological changes are also evident in these epithelia (Nii et al., 1964; Lightwood and Nolan, 1970; Olding-Stenkvist and Bjorvatn, 1976; Moench et al., 1988; Craighead, 2000; Figure 1). In 2003, primary cultures of human small airway epithelial cells (SAECs) were shown to be susceptible to wt MV infection (Takeuchi et al., 2003). Upon MV infection, large syncytia developed in SAECs via a SLAM-independent mechanism (Takeuchi et al., 2003). After searching many cell lines, several epithelial cell lines with high susceptibility to MV infection were identified (Takeda et al., 2007; Tahara et al., 2008). Tahara et al. (2008) and Leonard et al. (2008) clearly demonstrated that wt MV infects epithelial cell lines that form tight junctions (TJs) using an unidentified receptor (Leonard et al., 2008; Tahara et al., 2008). Using these cells and recombinant IC323 expressing green fluorescent protein (IC323-EGFP; Takeda et al., 2000; Hashimoto et al., 2002), a final answer was obtained for the receptor on epithelial cells. Two groups independently demonstrated that nectin4, which is expressed at adherens junctions (AJs), acts as a receptor for MV (Mühlebach et al., 2011; Noyce et al., 2011). Interestingly, MV loses the ability to use either SLAM/CD150 or nectin4 when it possesses specific mutations in the $\mathrm{H}$ protein (Leonard et al., 2008; Tahara et al., 2008; Figure 2). Nectin4-blind MV still infects SLAM/CD150-positive immune cells efficiently (SLAM/CD150-tropic), and conversely, SLAM/CD150-blind MV infects nectin4-positive epithelial cells efficiently (nectin4-tropic; Leonard et al., 2008; Tahara et al., 2008; Figure 2). In this regard, MV is intrinsically dual-tropic to immune cells and epithelial cells (Figure 2).

There is now no doubt that SLAM/CD150 and nectin4 are the major receptors for MV. However, other molecules may further support MV infection in vivo, being involved in the development of measles and its neurological sequela. For example, the mechanism that the C-type lectin DC-specific intercellular adhesion

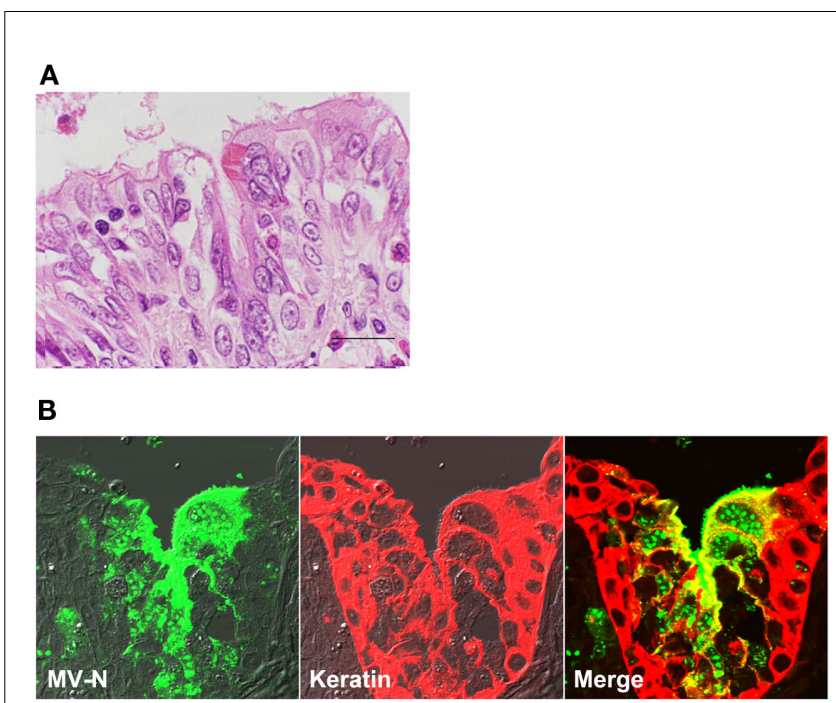

FIGURE 1 | Measles virus antigen and histopathological changes in bronchiolar epithelial cells. At 11 days post-infection, a cynomolgus monkey experimentally infected with wt MV IC323 strain was subjected to the histopathological examination. (A) Hematoxylin and eosin staining. Multinucleated cell were seen in the epithelial layer of the bronchiole with inflammatory infiltration of eosinophils and lymphocytes. The multinucleated cells contain eosinophilic intranuclear inclusions. Scale bar indicates $20 \mu \mathrm{m}$. (B) Immunohistochemical double staining for the $\mathrm{N}$ protein of MV (left, green) and keratin (center, red). Multinucleated epithelial giant cells showing positive staining for the $\mathrm{N}$ protein are present in the bronchiole (right, yellow in merged images). Accumulation of $\mathrm{N}$ proteins is detected on the apical surface of ciliated epithelial cells.

molecule 3-grabbing non-integrin (DC-SIGN) acts as an attachment receptor for MV, thereby promoting MV infection of DCs, may be ideal to understand the extraordinarily high transmissibility of measles (de Witte et al., 2006, 2008). It is well known that MV causes subacute sclerosing panencephalitis (SSPE), a persistent infection of the central nervous system (CNS) with MV. This occurs with a mean latency period of 7-10 years after suffering from acute measles at a frequency of $1 / 5,000-1 / 100,000$ reported cases of acute measles (Takasu et al., 2003; Bellini et al., 2005). The mechanisms underlying the spread of MV in the CNS remain to be elucidated. Although nectin4 is a possible candidate for an MV receptor in the CNS, no (or undetectable) nectin4 expression was observed in the CNS in humans (Reymond et al., 2001; Brancati et al., 2010), and some MV strains derived from SSPE patients are likely to use nectin4 inefficiently (Seki et al., 2011). Data reported by Makhortova et al. (2007) suggest that neurokinin-1, a substance $\mathrm{P}$ receptor, supports trans-synaptic transmission of MV by acting as a receptor for the F protein.

\section{SLAM/CD150}

Measles virus infection causes immunosuppression in patients and is often accompanied by secondary bacterial infections. Typically, $\mathrm{MV}$-induced immunosuppression is characterized by a marked lymphopenia, and an early $\mathrm{T}_{\mathrm{H}} 1$ response followed by predominant and prolonged $\mathrm{T}_{\mathrm{H}} 2$ response in patients, with suppression of mitogen-induced lymphocyte proliferation ex vivo (Griffin and 


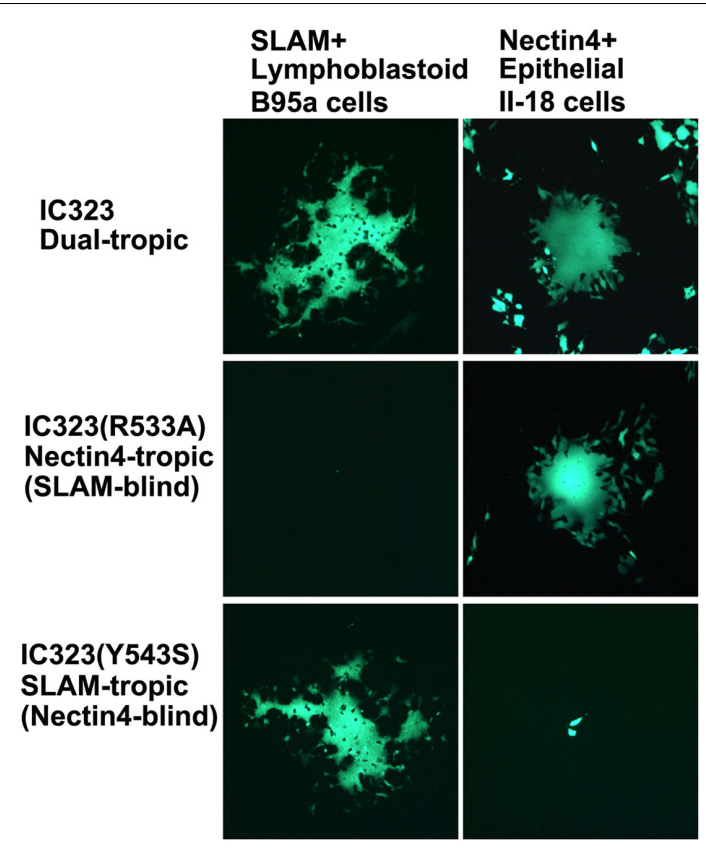

FIGURE 2 | Dual-tropic nature of wt MV. Wt MV (IC323) infects and produces syncytia in both SLAM-positive lymphoblastoid B95a and nectin4-positive epithelial II-18 cells (dual-tropic). IC323 with an R533A mutation in the $\mathrm{H}$ protein fails to spread in B95a cells (SLAM-blind), but replicates well in II-18 cells (nectin4-tropic). On the contrary, IC323 with a Y543S mutation in the $\mathrm{H}$ protein spreads in B95a cells (SLAM-tropic), but fails to spread in II-18 cells (nectin4-blind). All MVs in this figure are engineered to express EGFP.

Ward, 1993; Schneider-Schaulies and Schneider-Schaulies, 2009). Some, if not all, of these immunological observations must be attributed either directly or indirectly to the fact that MV uses SLAM/CD150 as a receptor. SLAM/CD150 is a member of the SLAM-family receptors, which belong to the immunoglobulin (Ig) superfamily (Veillette, 2010; Ma and Deenick, 2011). The SLAM-family consists of nine members (Cannons et al., 2011; Ma and Deenick, 2011). The SLAM-family receptors are type I transmembrane proteins that typically possess an extracellular region with two Ig-like domains (an amino-terminal variable (V)-like domain and a carboxy-terminal constant-2 (C2)-like domain), a transmembrane region, and a cytoplasmic region that harbors multiple tyrosine-based motifs (Detre et al., 2010; Veillette, 2010; Cannons et al., 2011; Ma and Deenick, 2011). These motifs are referred to as immunoreceptor tyrosine-based switch motifs (ITSMs; Cannons et al., 2011). The SLAM-family receptors are expressed in a broad range of immune cells and play critical roles in immunity. In general, the receptors act as selfligands and their homophilic trans-interactions occur between either heterotypic or homotypic immune cells (Veillette, 2010; Ma and Deenick, 2011). SLAM/CD150 is expressed on thymocytes, subsets of B and T lymphocytes, mature dendritic cells (DCs), macrophages, and platelets, and their expression is upregulated or induced in lymphocytes and monocytes upon activation (Detre et al., 2010; Veillette, 2010; Cannons et al., 2011; Ma and Deenick, 2011).
Signaling lymphocyte activation molecule-associated protein (SAP)-family adaptors [SAP, Ewing's sarcoma-associated transcript (EAT)-2, and EAT-2-related transducer (ERT)] play important roles for the signal transductions mediated by the SLAM-family receptors (Veillette, 2010; Ma and Deenick, 2011). They are small proteins that consist of a single Src homology 2 (SH2) domain and a short carboxy-terminal region. SAP associates intracellularly with the ITSMs in the cytoplasmic region of the SLAM-family receptors via the SH2 domain (Dong and Veillette, 2010; Veillette, 2010; Ma and Deenick, 2011). SAP has the ability to bind concomitantly to the Src-family protein tyrosine kinase, Fyn, thereby coupling the SLAM-family receptors with Fyn (Dong and Veillette, 2010; Veillette, 2010; Cannons et al., 2011). Thereafter, Fyn phosphorylates tyrosine residues at the cytoplasmic region of SLAM-family receptors and other intracellular effector molecules, activating the downstream signals (Detre et al., 2010; Dong and Veillette, 2010; Cannons et al., 2011; Ma and Deenick, 2011). In another mechanism, SAP binding to the $\mathrm{SH} 2$ domain of SLAM-family receptors competes with the binding of other $\mathrm{SH} 2$ domain-containing molecules, thus modulating the SLAM-mediated signaling (Dong and Veillette, 2010; Veillette, 2010; Cannons et al., 2011). In CD4 ${ }^{+} \mathrm{T}$ cells, signals via SLAM/CD150-SAP-Fyn interactions play important roles in regulating $\mathrm{T}$ cell receptor-mediated induction of $\mathrm{T}_{\mathrm{H}} 2$ cytokines, such as interleukin (IL)-4 and IL-13 (Detre et al., 2010; Cannons et al., 2011; Ma and Deenick, 2011). EAT-2 also mediates the signal transduction cascades of the SLAM-family receptors via a similar but distinct mechanism to that of SAP (Cannons et al., 2011). Similar to SAP, EAT-2 also associates with the ITSMs of SLAM-family receptors through its $\mathrm{SH} 2$ domain, but mediates the subsequent signal cascades via its own phosphorylated tyrosine in the short carboxy-terminal region (Veillette, 2010). In general, the signals mediated by the SAP-family adaptors induce the activation and differentiation of immune cells (Veillette, 2010). However, if the SAP-family adaptors are absent, the SLAM-family receptors mediate inhibitory signals to immune cells (a switch-of-function effect; Dong and Veillette, 2010; Veillette, 2010).

Roles for SLAM/CD150 in macrophage functions, cell adhesion, and NKT cell development have also been demonstrated, although many data were obtained in mice (Dong and Veillette, 2010; Veillette, 2010; Cannons et al., 2011; Ma and Deenick, 2011). $\mathrm{X}$-linked lymphoproliferative syndrome is a rare immunodeficiency disease typically caused by mutations in the SAP-encoding gene, SH2D1A (Veillette, 2010; Ma and Deenick, 2011). Patients with this syndrome have various functional defects and impaired differentiation of immune cells, indicating crucial roles for SAP in normal immunity (Dong and Veillette, 2010). Hypogammaglobulinemia, massive lymphoproliferative syndrome, and a fatal response to Epstein-Barr virus infection are characteristics of the disease (Veillette, 2010; Ma and Deenick, 2011). Although SLAMfamily receptors have some functional redundancy, each receptor plays specific roles in a variety of immune responses (Dong and Veillette, 2010; Veillette, 2010).

\section{NECTIN4}

In general, lymphotropic viruses, such as HIV and HTLV1, can never be airborne, and are transmitted inefficiently even through 
direct contact with patients. In sharp contrast, MV transmits via aerosols, and has a highly contagious nature. Therefore, this transmission style of MV cannot be easily explained by the fact that the virus uses a lymphocytic molecule, SLAM/CD150, as a receptor. The recent findings showing that MV uses nectin4, a cell adhesion molecule (CAM) expressed at the AJs of epithelia, may partly but nicely explain how and why MV transmits efficiently from a patient to other individuals. Epithelial cells are connected with one another through the formation of several specialized cell-cell junctions, such as TJs, AJs, desmosomes, and gap junctions. TJs function as a physical barrier that prevents the passage of soluble molecules through the intercellular gaps, and also blocks the lateral movement of lipids and membrane proteins across the TJ barrier, thereby acting as the border of the apical and basolateral membranes. AJs are located near the basolateral side of the TJs. They are basically formed by cadherins and nectins and intracellularly connected by actin filaments. Nectin 4 is a member of the nectin family, which consists of four members (nectin 1, 2, 3, and 4; Takai et al., 2008a). Nectin1 and nectin2 were originally identified as poliovirus receptor-related protein (PRR)-1 and PRR-2, respectively, and subsequently shown to support the entry of some herpes viruses (Takai et al., 2008a,b). Similar to the SLAM-family members, nectins are also type I transmembrane proteins that belong to the Ig superfamily (Takai et al., 2008a,b). In general, they possess an extracellular region with three Ig-like domains (an amino-terminal V-like domain and two C-like domains), a transmembrane region, and a cytoplasmic region with a short afadinbinding motif (Takai et al., 2008a,b). The consensus motif was reported to be E/A-X-Y-V for nectin 1, 2, and 3, while nectin4 does not have this motif but still binds to afadin (Reymond et al., 2001; Takai et al., 2008a). Reymond et al. (2001) proposed a new consensus motif, K/R-X-X-Y/L-V, for all four nectins. Afadin is an actin filament (F-actin)-binding protein, and supports nectins to interact and co-operate with cadherins, other CAMs, and intracellular signaling molecules (Takai et al., 2008a).

Nectins are expressed as dimers, and interact in trans with other nectin dimers expressed on neighboring cells (Takai et al., 2008a,b). All nectins show homophilic interactions, while heterophilic interactions are also observed between specific nectins, such as those between nectin $1 /$ nectin 3 and nectin 2/nectin3 (Takai et al., 2008a,b). Some nectin-like molecules also interact with nectins (Takai et al., 2008a,b). Nectin4 shows homophilic interactions as well as heterophilic interactions with nectin1 (Reymond et al., 2001; Takai et al., 2008a). The Ig V-like domain is used for the trans-interaction (Reymond et al., 2001; Fabre et al., 2002). Nectins play key roles in the initiation of AJ formation, and regulate various physiological functions of epithelial cells, such as contact inhibition of cell movement and proliferation, survival, differentiation, and cell polarization (Takai et al., 2008a,b).

Although nectin 1 and nectin 2 are expressed in a broad range of tissues, the expression of nectin 3 and nectin 4 is more specific (Reymond et al., 2001). Reymond et al. (2001) and Brancati et al. (2010) showed that human nectin4 is expressed mainly in the placenta and to lesser extents in the trachea, prostate, lung, and stomach. In addition, Brancati et al. (2010) demonstrated nectin4 expression in human keratinocytes, suprabasal nucleated layers of the epidermis, and non-keratinized structures of hair. Some levels of expression in epithelial cells of the tonsil, oral mucosa, esophagus, and nasopharynx have also been reported (www.proteinatlas.org). Although, in many cases, nectin4 is expressed in low or undetectable levels in normal human tissues, many cancer cells are highly positive for nectin 4 . Thus, it has been proposed that nectin 4 is a new tumor-associated marker (FabreLafay et al., 2007; Takano et al., 2009; Derycke et al., 2010). These observations may provide a rationale for the use of $\mathrm{MV}$ as an oncolytic agent (Mühlebach et al., 2011). In humans, mutations in the PVRL4 gene encoding nectin4 cause ectodermal-dysplasiasyndactyly syndrome (EDSS), in which patients have affected skin and skin appendages, such as hair, teeth, and nails (Brancati et al., 2010; Jelani et al., 2011).

\section{RELEVANCE OF SLAM/CD150 AND NECTIN4 TO MV PATHOGENESIS}

The pathology of measles can now be drawn with these two receptors (Figure 3). Although nectin4-expressing epithelial cells can be the initial targets of MV, no or very limited infection of epithelia was observed in monkeys experimentally infected with MV at the early days after infection (Ludlow et al., 2010; Lemon et al., 2011). Instead, MV initiates its infection via SLAM-mediated entry into alveolar macrophages and DCs in the lung or respiratory tracts (de Witte et al., 2008; de Vries et al., 2010; Lemon et al., 2011). These infections may allow MV to penetrate into the human body and reach the lymphoid organs or tissues, where SLAM/CD150expressing cells are abundant (Corry et al., 1984; Lehmann et al., 2001). After initial replication in these lymphoid organs or tissues, MV or MV-infected lymphocytes can easily enter the bloodstream. Subsequently, a dramatic MV infection is observed in all lymphoid organs, such as the spleen, thymus, appendix, tonsil, and lymph nodes, throughout the body (Moench et al., 1988; Kobune et al., 1996; de Swart et al., 2007; de Vries et al., 2010). At the time when the MV infection of lymphoid organs reaches its peak, MV infection of epithelia, such as squamous stratified epithelia of the tongue and buccal mucosa and ciliated epithelia of the trachea, becomes evident (Nii et al., 1964; Olding-Stenkvist and Bjorvatn, 1976; Moench et al., 1988; de Swart et al., 2007). This epithelial infection is probably led by MV-infected immune cells and initiated through the basolateral side, since monkeys infected with MV often show infectious foci in the epithelia with MVinfected lymphoid or myeloid cells in the subepithelial cell layers of the trachea, bronchus, and tongue (de Vries et al., 2010; Ludlow et al., 2010). The $\mathrm{H}$ protein expressed on MV-infected immune cells that migrate through the epithelial cell layer likely recognizes nectin4 expressed at AJs, triggering $\mathrm{F}$ protein-mediated membrane fusion between the MV-infected immune cells and the target epithelial cells. Mühlebach et al. (2011) demonstrated a correlation between nectin4 expression and MV infection in epithelia in vivo. Importantly, MV has a mechanism that further facilitates virus shedding in the airway. In epithelia, MV selectively releases progeny virus particles from the apical membrane to the luminal side of the respiratory tract (Leonard et al., 2008; Tahara et al., 2008). Leonard et al. (2008) showed that MV genetically engineered to use SLAM/CD150, but not nectin4 (nectin4-blind or SLAM/CD150tropic), does not shed progeny viruses into the respiratory tract, 


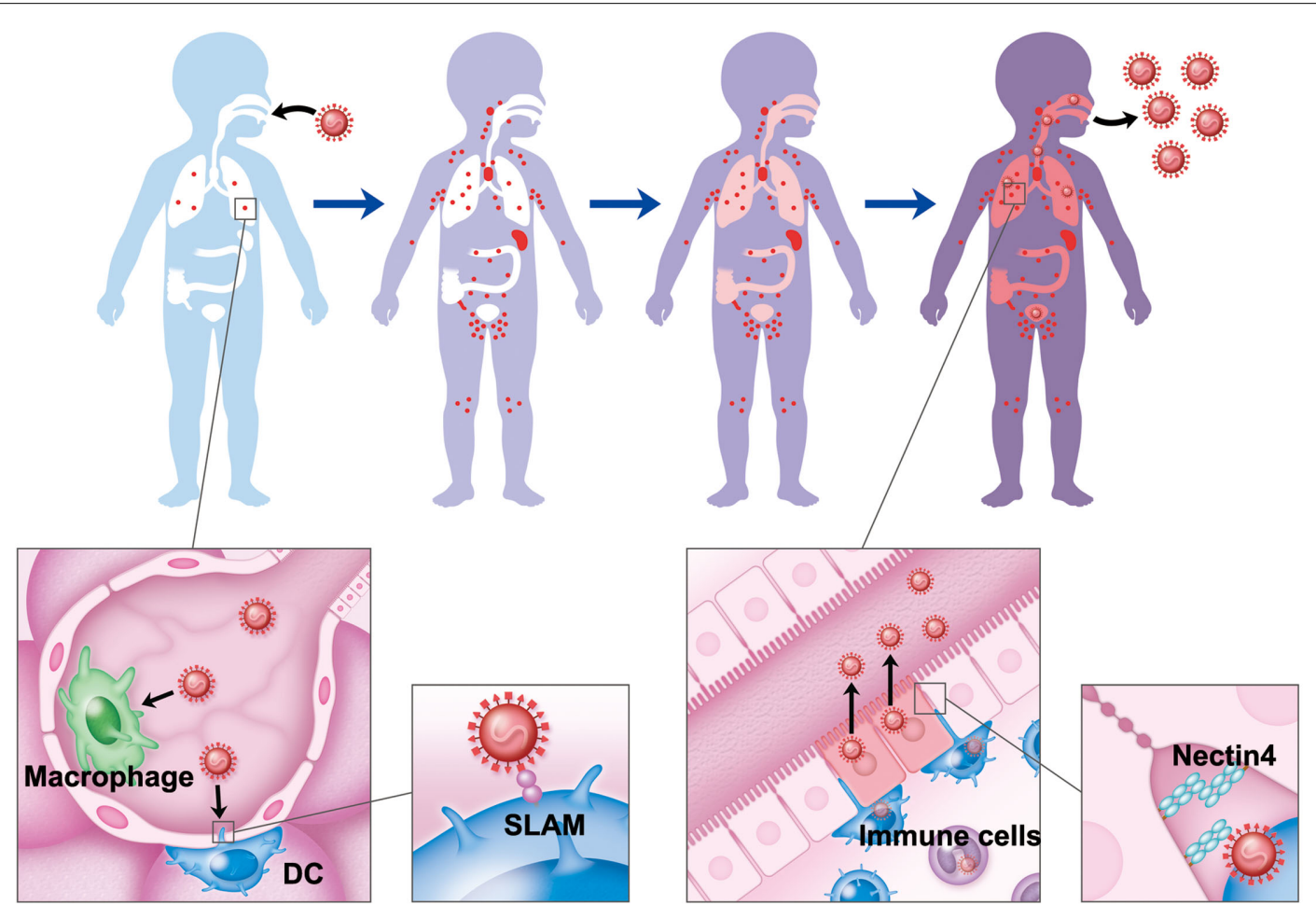

FIGURE 3 | Pathology of measles. MV initiates its infection via SLAM-mediated entry into alveolar macrophages and DCs in the lung. After initial replication in regional lymph nodes, MV enters the bloodstream and subsequently spreads to all lymphoid organs, such as the spleen, thymus, appendix, tonsil, and lymph nodes, throughout the body. When the MV infection of the lymphoid organs reaches its peak, MV-infected immune cells transfer MV to epithelial cells through the basolateral side. At this step, MV uses nectin4 expressed at AJs as a receptor. In the epithelia, MV selectively releases its progeny virus particles from the apical membrane to the luminal side of the respiratory tract. although it does show systemic infection of lymphoid organs, similar to the case for wt MV.

\section{CONCLUDING REMARKS}

Membrane cofactor protein/CD46 was first identified as a receptor for MV (Dorig et al., 1993; Naniche et al., 1993). However, our current knowledge of MV receptors has been totally transformed. In 2000, it was shown that SLAM/CD150 expressed on cells of the immune system, but not MCP/CD46, is a real receptor for wt MV. However, recent studies further showed that SLAM/CD150 is not the sole receptor for MV. MV has an intrinsic ability to enter not

\section{REFERENCES}

Bellini, W. J., Rota, J. S., Lowe, L. E., Katz, R. S., Dyken, P. R., Zaki, S. R., Shieh, W. J., and Rota, P. A. (2005). Subacute sclerosing panencephalitis: more cases of this fatal disease are prevented by measles immunization than was previously recognized. J. Infect. Dis. 192, 1686-1693.

Brancati, F., Fortugno, P., Bottillo, I., Lopez, M., Josselin, E., BoudgheneStambouli, O., Agolini, E., Bernardini, L., Bellacchio, E., Iannicelli, M., Rossi, A., Dib-Lachachi, A., Stuppia, L., Palka, G., Mundlos, S., Stricker,
S., Kornak, U., Zambruno, G., and Dallapiccola, B. (2010). Mutations in PVRL4, encoding cell adhesion molecule nectin- 4 , cause ectodermal dysplasia-syndactyly syndrome. Am. J. Hum. Genet. 87, 265-273.

Cannons, J. L., Tangye, S. G., and Schwartzberg, P. L. (2011). SLAM family receptors and SAP adaptors in immunity. Annu. Rev. Immunol. 29, 665-705.

Corry, D., Kulkarni, P., and Lipscomb, M. F. (1984). The migration of bronchoalveolar macrophages into hilar lymph nodes. Am. J. Pathol. 115, 321-328.

only immune cells but also epithelial cells. In 2011, a clear answer was obtained through the identification of the epithelial MV receptor nectin4, which is expressed at AJs, thereby partly explaining why MV exhibits its highly contagious nature (Mühlebach et al., 2011; Noyce et al., 2011). Recent studies on MV receptors greatly advanced our understanding of MV pathogenesis. However, many aspects and molecular mechanisms underlying immunosuppressive effects and a highly contagious nature of MV still remain to be elucidated. Analyses of physiological roles of MV receptors, SLAM/CD150, and nectin4, would provide deep insights into MV pathogenesis.

Craighead, J. E. (2000). "Rubeola (Measles)," in Pathology and Pathogenesis of Human Viral Disease, ed. J. E. Craighead (Philadelphia, PA: Elsevier Inc.), 397-410.

de Swart, R. L., Ludlow, M., De Witte, L., Yanagi, Y., Van Amerongen, G., Mcquaid, S., Yuksel, S., Geijtenbeek, T. B., Duprex, W. P., and Osterhaus, A. D. (2007). Predominant infection of CD150(+) lymphocytes and dendritic cells during measles virus infection of macaques. PLoS Pathog. 3, e178. doi: 10.1371/journal.ppat. 0030178 de Vries, R. D., Lemon, K., Ludlow, M., Mcquaid, S., Yuksel, S., Van Amerongen, G., Rennick, L. J., Rima, B. K., Osterhaus, A. D., De Swart, R. L., and Duprex, W. P. (2010). In vivo tropism of attenuated and pathogenic measles virus expressing green fluorescent protein in macaques. J. Virol. 84, 4714-4724.

de Witte, L., Abt, M., SchneiderSchaulies, S., Van Kooyk, Y., and Geijtenbeek, T. B. (2006). Measles virus targets DC-SIGN to enhance dendritic cell infection. J. Virol. 80 , 3477-3486 
de Witte, L., De Vries, R. D., Van Der Vlist, M., Yuksel, S., Litjens, M., De Swart, R. L., and Geijtenbeek, T. B. (2008). DC-SIGN and CD150 have distinct roles in transmission of measles virus from dendritic cells to T-lymphocytes. PLoS Pathog. 4, el000049. doi:10.1371/journal.ppat.1000049

Derycke, M. S., Pambuccian, S. E., Gilks, C. B., Kalloger, S. E., Ghidouche, A., Lopez, M., Bliss, R. L., Geller, M. A., Argenta, P. A., Harrington, K. M., and Skubitz, A. P. (2010). Nectin 4 overexpression in ovarian cancer tissues and serum: potential role as a serum biomarker. Am. J. Clin. Pathol. 134, 835-845.

Detre, C., Keszei, M., Romero, X., Tsokos, G. C., and Terhorst, C. (2010). SLAM family receptors and the SLAM-associated protein (SAP) modulate $\mathrm{T}$ cell functions. Semin. Immunopathol. 32, 157-171.

Devaux, P., Hodge, G., Mcchesney, M. B., and Cattaneo, R. (2008). Attenuation of $\mathrm{V}$ - or C-defective measles viruses: infection control by the inflammatory and interferon responses of rhesus monkeys. J. Virol. 82, 5359-5367.

Devaux, P., Hudacek, A. W., Hodge, G., Reyes-Del Valle, J., Mcchesney, M. B., and Cattaneo, R. (2011). A recombinant measles virus unable to antagonize STAT1 function cannot control inflammation and is attenuated in rhesus monkeys. J. Virol. 85, 348-356.

Dong, Z., and Veillette, A. (2010). How do SAP family deficiencies compromise immunity? Trends Immunol. 31, 295-302.

Dorig, R. E., Marcil, A., Chopra, A., and Richardson, C. D. (1993). The human CD46 molecule is a receptor for measles virus (Edmonston strain). Cell 75, 295-305.

Fabre, S., Reymond, N., Cocchi, F., Menotti, L., Dubreuil, P., Campadelli-Fiume, G., and Lopez, M. (2002). Prominent role of the Ig-like $\mathrm{V}$ domain in transinteractions of nectins. Nectin3 and nectin 4 bind to the predicted C-C'-C $C^{\prime \prime}-\mathrm{D}$ beta-strands of the nectin1 V domain. J. Biol. Chem. 277, 27006-27013.

Fabre-Lafay, S., Monville, F., GarridoUrbani, S., Berruyer-Pouyet, C., Ginestier, C., Reymond, N., Finetti, P., Sauvan, R., Adelaide, J., Geneix, J., Lecocq, E., Popovici, C., Dubreuil, P., Viens, P., Goncalves, A., CharafeJauffret, E., Jacquemier, J., Birnbaum, D., and Lopez, M. (2007). Nectin-4 is a new histological and serological tumor associated marker for breast cancer. BMC Cancer 7, 73 . doi:10.1186/1471-2407-7-73

Griffin, D. E. (2007). “Measles virus,” in Fields Virology, 5th Edn, eds D. M. Knipe, P. M. Howley, D. E. Griffin, R. A. Lamb, M. A. Martin, B. Roizman, and S. E. Straus (Philadelphia: Lippincott Williams and Wilkins), 1551-1585.

Griffin, D. E., and Ward, B. J. (1993). Differential CD4 $\mathrm{T}$ cell activation in measles. J. Infect. Dis. 168, 275-281.

Hashimoto, K., Ono, N., Tatsuo, H., Minagawa, H., Takeda, M., Takeuchi, K., and Yanagi, Y. (2002). SLAM (CD150)-independent measles virus entry as revealed by recombinant virus expressing green fluorescent protein. J. Virol. 76, 6743-6749.

Jelani, M., Chishti, M. S., and Ahmad, W. (2011). Mutation in PVRL4 gene encoding nectin-4 underlies ectodermal-dysplasia-syndactyly syndrome (EDSS1). J. Hum. Genet. 56, 352-357.

Kobune, F., Sakata, H., and Sugiura, A. (1990). Marmoset lymphoblastoid cells as a sensitive host for isolation of measles virus. J. Virol. 64, 700-705.

Kobune, F., Takahashi, H., Terao, K., Ohkawa, T., Ami, Y., Suzaki, Y., Nagata, N., Sakata, H., Yamanouchi, K., and Kai, C. (1996). Nonhuman primate models of measles. Lab. Anim. Sci. 46, 315-320.

Lehmann, C., Wilkening, A., Leiber, D., Markus, A., Krug, N., Pabst, R., and Tschernig, T. (2001). Lymphocytes in the bronchoalveolar space reenter the lung tissue by means of the alveolar epithelium, migrate to regional lymph nodes, and subsequently rejoin the systemic immune system. Anat. Rec. 264, 229-236.

Lemon, K., De Vries, R. D., Mesman, A. W., Mcquaid, S., Van Amerongen, G., Yuksel, S., Ludlow, M., Rennick, L. J., Kuiken, T., Rima, B. K., Geijtenbeek, T. B., Osterhaus, A. D., Duprex, W. P., and De Swart, R. L. (2011). Early target cells of measles virus after aerosol infection of non-human primates. PLoS Pathog. 7, e1001263. doi:10.1371/journal.ppat.1001263

Leonard, V. H., Sinn, P. L., Hodge, G., Miest, T., Devaux, P., Oezguen, N., Braun, W., Mccray, P. B., Mcchesney, M. B., and Cattaneo, R. (2008). Measles virus blind to its epithelial cell receptor remains virulent in rhesus monkeys but cannot cross the airway epithelium and is not shed. J. Clin. Invest. 118, 2448-2458.

Lightwood, R., and Nolan, R. (1970). Epithelial giant cells in measles as an acid in diagnosis. J. Pediatr. 77, 59-64.
Ludlow, M., Rennick, L. J., Sarlang, S. Skibinski, G., Mcquaid, S., Moore, T., De Swart, R. L., and Duprex, W. P. (2010). Wild-type measles virus infection of primary epithelial cells occurs via the basolateral surface without syncytium formation or release of infectious virus. J. Gen. Virol. 91, 971-979.

Ma, C. S., and Deenick, E. K. (2011). The role of SAP and SLAM family molecules in the humoral immune response. Ann. N. Y. Acad. Sci. 1217, 32-44.

Makhortova, N. R., Askovich, P., Patterson, C. E., Gechman, L. A., Gerard, N. P., and Rall, G. F. (2007). Neurokinin-1 enables measles virus trans-synaptic spread in neurons. Virology 362, 235-244.

Moench, T. R., Griffin, D. E., Obriecht, C. R., Vaisberg, A. J., and Johnson, R. T. (1988). Acute measles in patients with and without neurological involvement: distribution of measles virus antigen and RNA. J. Infect. Dis. 158, 433-442.

Mühlebach, M. D., Mateo, M., Sinn, P. L., Prufer, S., Uhlig, K. M., Leonard, V. H., Navaratnarajah, C. K., Frenzke, M., Wong, X. X., Sawatsky, B., Ramachandran, S., Mccray, P. B., Cichutek, K., Von Messling, V., Lopez, M., and Cattaneo, R. (2011). Adherens junction protein nectin-4 is the epithelial receptor for measles virus. Nature 480, 530-533.

Nakatsu, Y., Takeda, M., Ohno, S., Shirogane, Y., Iwasaki, M., and Yanagi, Y. (2008). Measles virus circumvents the host interferon response by different actions of the $\mathrm{C}$ and $\mathrm{V}$ proteins. J. Virol. 82, 8296-8306.

Naniche, D., Varior-Krishnan, G., Cervoni, F., Wild, T. F., Rossi, B., Rabourdin-Combe, C., and Gerlier, D. (1993). Human membrane cofactor protein (CD46) acts as a cellular receptor for measles virus. J. Virol. 67, 6025-6032.

Nii, S., Kamahora, J., Mori, Y., Takahashi, M., Nishimura, S., and Okuno, Y. (1964). Experimental pathology of measles in monkeys. Biken J. 6, 271-297.

Noyce, R. S., Bondre, D. G., Ha, M. N., Lin, L. T., Sisson, G., Tsao, M. S., and Richardson, C. D. (2011). Tumor cell marker PVRL4 (nectin 4) is an epithelial cell receptor for measles virus. PLoS Pathog. 7, e1002240. doi:10.1371/journal.ppat.1002240

Olding-Stenkvist, E., and Bjorvatn, B. (1976). Rapid detection of measles virus in skin rashes by immunofluroescence. J. Infect. Dis. 134, 463-469.
Reymond, N., Fabre, S., Lecocq, E., Adelaide, J., Dubreuil, P., and Lopez, M. (2001). Nectin4/PRR4, a new afadinassociated member of the nectin family that trans-interacts with nectin1/PRR1 through $\mathrm{V}$ domain interaction. J. Biol. Chem. 276, 43205-43215.

Schneider-Schaulies, S., and SchneiderSchaulies, J. (2009). Measles virus-induced immunosuppression. Curr. Top. Microbiol. Immunol. 330, 243-269.

Seki, F., Yamada, K., Nakatsu, Y., Okamura, K., Yanagi, Y., Nakayama, T., Komase, K., and Takeda, M. (2011). The si strain of measles virus derived from a patient with subacute sclerosing panencephalitis possesses typical genome alterations and unique amino acid changes that modulate receptor specificity and reduce membrane fusion activity. J. Virol. 85, 11871-11882

Tahara, M., Takeda, M., Shirogane, Y., Hashiguchi, T., Ohno, S., and Yanagi, Y. (2008). Measles virus infects both polarized epithelial and immune cells by using distinctive receptor-binding sites on its hemagglutinin. J. Virol. 82, 4630-4637.

Takai, Y., Ikeda, W., Ogita, H., and Rikitake, Y. (2008a). The immunoglobulin-like cell adhesion molecule nectin and its associated protein afadin. Annu. Rev. Cell Dev. Biol. 24, 309-342.

Takai, Y., Miyoshi, J., Ikeda, W., and Ogita, H. (2008b). Nectins and nectin-like molecules: roles in contact inhibition of cell movement and proliferation. Nat. Rev. Mol. Cell Biol. 9, 603-615.

Takano, A., Ishikawa, N., Nishino, R., Masuda, K., Yasui, W., Inai, K., Nishimura, H., Ito, H., Nakayama, H., Miyagi, Y., Tsuchiya, E., Kohno, N., Nakamura, Y., and Daigo, Y. (2009). Identification of nectin-4 oncoprotein as a diagnostic and therapeutic target for lung cancer. Cancer Res. 69, 6694-6703.

Takasu, T., Mgone, J. M., Mgone, C. S., Miki, K., Komase, K., Namae, H., Saito, Y., Kokubun, Y., Nishimura, T., Kawanishi, R., Mizutani, T., Markus, T. J., Kono, J., Asuo, P. G., and Alpers, M. P. (2003). A continuing high incidence of subacute sclerosing panencephalitis (SSPE) in the Eastern Highlands of Papua New Guinea. Epidemiol. Infect. 131, 887-898.

Takeda, M., Kato, A., Kobune, F., Sakata, H., Li, Y., Shioda, T., Sakai, Y., Asakawa, M., and Nagai, Y. (1998). 
Measles virus attenuation associated with transcriptional impediment and a few amino acid changes in the polymerase and accessory proteins. J. Virol. 72, 8690-8696.

Takeda, M., Tahara, M., Hashiguchi, T., Sato, T. A., Jinnouchi, F., Ueki, S., Ohno, S., and Yanagi, Y. (2007). A human lung carcinoma cell line supports efficient measles virus growth and syncytium formation via a SLAM- and CD46-independent mechanism. J. Virol. 81, 12091-12096.

Takeda, M., Takeuchi, K., Miyajima, N., Kobune, F., Ami, Y., Nagata, N., Suzaki, Y., Nagai, Y., and Tashiro, M. (2000). Recovery of pathogenic measles virus from cloned cDNA. J. Virol. 74, 6643-6647.
Takeuchi, K., Miyajima, N., Nagata, N., Takeda, M., and Tashiro, M. (2003). Wild-type measles virus induces large syncytium formation in primary human small airway epithelial cells by a SLAM(CD150)independent mechanism. Virus Res. 94, 11-16.

Takeuchi, K., Takeda, M., Miyajima, N., Ami, Y., Nagata, N., Suzaki, Y., Shahnewaz, J., Kadota, S., and Nagata, K. (2005). Stringent requirement for the C protein of wildtype measles virus for growth both in vitro and in macaques. J. Virol. 79, 7838-7844.

Tatsuo, H., Ono, N., Tanaka, K., and Yanagi, Y. (2000). SLAM (CDw150) is a cellular receptor for measles virus. Nature 406, 893-897.
Veillette, A. (2010). SLAM-family receptors: immune regulators with or without SAP-family adaptors. Cold Spring Harb. Perspect. Biol. 2, a002469.

WHO. (2009). Global reductions in measles mortality 2000-2008 and the risk of measles resurgence. Wkly. Epidemiol. Rec. 84, 509-516.

Yanagi, Y., Takeda, M., Ohno, S., and Hashiguchi, T. (2009). Measles virus receptors. Curr. Top. Microbiol. Immunol. 329, 13-30.

Conflict of Interest Statement: The authors declare that the research was conducted in the absence of any commercial or financial relationships that could be construed as a potential conflict of interest.
Received: 15 November 2011; paper pending published: 26 November 2011; accepted: 26 December 2011; published online: 13 January 2012.

Citation: Takeda M, Tahara M, Nagata $N$ and Seki F (2012) Wild-type measles virus is intrinsically dualtropic. Front. Microbio. 2:279. doi: 10.3389/fmicb.2011.00279

This article was submitted to Frontiers in Virology, a specialty of Frontiers in Microbiology.

Copyright (C) 2012 Takeda, Tahara, Nagata and Seki. This is an open-access article distributed under the terms of the Creative Commons Attribution Non Commercial License, which permits noncommercial use, distribution, and reproduction in other forums, provided the original authors and source are credited. 